\title{
Identificación de las barreras de acceso a la información, a la participación y al aprendizaje en el Museo Es Baluard
}

\author{
BEgOÑA DE LA IGLESIA-MAYOL \\ Universitat de les Islles Balears \\ bego.delaiglesia@uib.es \\ MARÍA Rosa Rosselló-RAMÓN \\ Universitat de les Islles Balears \\ mrosa.rossello@uib.es
}

Recibido: 03 de octubre de 2012

Aceptado: 22 de marzo de 2013

\section{Resumen}

El artículo presenta una investigación ${ }^{1}$ colaborativa desarrollada entre el GREID (Grupo de Investigación sobre Escuela Inclusiva y Diversidad, de la UIB) y el Área de Educación de Es Baluard Museo de Arte Moderno y Contemporáneo de Palma. Se trata de un estudio pionero en el que se aplican los principios del diseño universal para valorar la accesibilidad que presenta el museo. En concreto, en el estudio se desarrollan dos objetivos básicos: primero, se identifican las barreras de acceso al edificio, a la información y a los contenidos que el museo ofrece a cualquier visitante. Segundo, se evalúa la accesibilidad de las visitas y talleres que se destinan a las escuelas. Mediante un diseño de investigación complejo que utiliza diversos instrumentos de recogida de información (observación directa, análisis y registro en vídeo de las sesiones, análisis documental y grupos de discusión), se detectan importantes barreras que reflejan una concepción homogénea de entender al visitante, aún predominante en el Museo y aún presente centros educativos.

Palabras clave: Museo de Arte; Escuela; Calidad de Vida; Diseño Universal; Inclusión.

De la Iglesia Mayol, B. Roselló Ramón, M (2013) Identificación de las barreras de acceso a la información, a la participación y al aprendizaje en el Museo Es Baluard, 26 (1) 21-38

\begin{abstract}
The paper presents a collaborative research between GREID (Research Group on Inclusive Education and Diversity, UIB) and the AEAC (Area of Education of Es Baluard, Museum of Modern and Contemporary Art of Palma).

This is a pioneering study that applies the principles of Universal Design to assess the accessibility that presents the museum. Specifically, the study suggests two basic objectives: The first one is to identify the barriers to undestand the information and content that the museum offers any visitor. The second one is to evaluate the accessibility of visits and workshops for schools. Through various data collection instruments (direct observation, analysis and video recordings of meetings, document analysis and focus groups), we identify significant barriers that reflect an homogeneus concepto $f$ visitor, still predominant in the Museum and also that is shared with a lot of schools.
\end{abstract}

Key words: Art Museum, School, Quality of life; Universal Design; Inclusion.

De la Iglesia Mayol, B. Roselló Ramón, M (2013) Identifying barriers to access to information, participation and learning in the Es Baluard Museum, 26 (1) 21-38 


\section{Antecedentes y planteamiento del tema}

Durante estos últimos 20 años y gracias a corrientes como la denominada Museología Crítica (Hernández, 2006; Padró, 2003; Santacana y Hernández, 2006), los museos han cambiado su centro de interés. Han pasado de centrarse en las obras de arte a intentar captar nuevos segmentos de público, buscando nuevas fórmulas comunicativas y didácticas. Vamos a una exposición y es habitual encontrar indicadores que nos facilitan el acceso y la interpretación de las obras (recursos audiovisuales, información multisensorial, guías informativas, etc). El interés se ha desplazado del objeto al visitante. Sin embargo, como tendremos ocasión de demostrar en este artículo, todavía no alcanza a todos los visitantes.

Desde su apertura en 2004, el Museo Es Baluard ha ido incorporando de manera progresiva estos cambios, asumiendo los principios de la museología y la pedagogía crítica. La coordinadora del Área de Educación y Acción Cultural del propio museo, lo explica del siguiente modo:

Es Baluard Museu d'Art Modern i Contemporani de Palma y su Área de Educación (AEAC) tienen como uno de sus principales objetivos acercar el arte contemporáneo a los diferentes públicos y colectivos, no sólo como finalidad en si misma sino también como medio para reflexionar sobre el mundo que nos rodea y desarrollar así el pensamiento y sentido crítico, a la vez que favorecer un aprendizaje significativo" (Cifre, 2011, 228) .

A través de sus diferentes programas el AEAC pretende:

- Acercar el arte contemporáneo a los diferentes públicos y colectivos, no sólo como finalidad en sí misma, sino también como medio para reflexionar sobre le mundo que nos rodea.

- Desarrollar el pensamiento y sentido crítico.

- Favorecer un aprendizaje significativo.

- Abrir el museo como un espacio de diálogo a la diversidad de colectivos que conforman la ciudadanía, para que así se convierta en un espacio público de la comunidad que favorezca la inclusión. (Cifre, 2011, p. 2)

Este último objetivo es el que lleva al AEAC a contactar con el GREID (Grup de Recerca en Escola Inclusiva i Diversitat) de la Universitat de les Illes Balears, ya que en las primeras experiencias educativas que el área diseña, los colectivos de personas con discapacidad o con enfermedades mentales, se ven excluidos. Aunque existe una tendencia a solucionar esta situación mediante talleres y visitas específicas para estos colectivos, buscan soluciones que permitan incrementar la accesibilidad al museo, a su entorno y a sus contenidos. Tras unos primeros contactos, el AEAC de Es Baluard y el GREID encuentran multitud de coincidencias en sus inquietudes y propuestas, detectándose un ámbito común de intervención: por una parte, el museo necesita revisar y mejorar la calidad y accesibilidad de sus programas educativos; por otra, los principios de la escuela inclusiva y del diseño universal de aprendizaje pueden ser 
un buen referente para este análisis, conectando directamente con las experiencias e investigaciones desarrolladas por el GREID. Surge así la posibilidad de constituir un equipo de investigación interdisciplinar, con el proyecto de aplicar el Diseño Universal a la oferta didáctica del museo, de manera que sea posible progresar hacia metas de mayor inclusión en este ámbito ${ }^{2}$.

\section{Fundamentos teóricos y conceptuales}

En la fundamentación del proyecto de investigación confluyen tres grandes referentes, o marcos teóricos de interpretación: 1. La Museología Crítica, como base de cambio en la función social y comunitaria de los museos. 2. El Diseño Universal, instrumento de análisis de la accesibilidad y 3. La Educación Inclusiva, marco de referencia para el planteamiento de cualquier propuesta de intervención.

1. En primer lugar, el cambio generalizado que sufren los museos en la manera de entender y concebir su función social y su papel en la comunidad.

Como otros museos, Es Baluard asume la urgencia de una apertura que transita en una doble dirección (hacia fuera/hacia dentro), para acoger cada vez más públicos, cada vez más diversos, plurales y heterogéneos. Como explica Martínez, los museos son espacios para el aprendizaje donde es posible "explorar, analizar, debatir, negociar, construir. Hacernos persona. Hacer mundo. Revelar la contingencia de las cosas. Vislumbrar la posibilidad de transformación de la realidad. Conducirla al descrédito. Propiciar procesos llenos de negociaciones, descubrimientos y cuestionamientos. Desarrollar prácticas que fomenten la discusión desde contextos sociales y políticos. Crear comunidades, generar asociaciones, establecer redes de cooperación, construir vías de conocimiento" (Martínez, 2008).

En estos últimos años, Es Baluard busca convertirse en un espacio capaz de impulsar y promover nuevas miradas a la sociedad, facilitando el intercambio de visiones y experiencias entre las obras y sus visitantes.

Tomando como punto de partida la misión de Es Baluard como servicio público y conscientes del creciente papel que están adquiriendo los museos como comunicadores socioculturales, convirtiéndose en un instrumento para el desarrollo de la sociedad en la que se encuentran inmersos, la intención del área es abrir el museo como un espacio de diálogo a la diversidad de colectivos que conforman la ciudadanía, para que así se convierta en un espacio que favorezca la inclusión. (Cifre, 2011, p. 2).

Pero, al recoger los planteamientos de la museología crítica, la institución no sólo amplia su manera de entender su cometido social, sino que, a la vez, revisa su manera de entender al visitante. Este cambio de mirada es muy similar al que viene experimentando la escuela al abandonar un modelo de enseñanza centrado exclusivamente en la transmisión de los conocimientos:

...el museo de arte contiene personas porque son quienes crean las obras, quienes las seleccionan, las exponen creando discursos y, al final, quienes las contemplan en los propios museos. Y, por todo ello, el museo potencialmente contiene experiencias, que precisamente viven personas. Todos estos contenidos nos sugieren un recorri- 
do que transita por lo conceptual, lo procedimental, lo actitudinal y lo experiencial. (Fontal, 2009, p. 89)

Sin embargo, como apunta Serrat (2008) la generación de nuevas ofertas educativas en los museos no siempre viene acompañada de una propuesta didáctica propia, coherente y bien fundamentada:

Frecuentemente se constata que la concepción didáctica en muchos museos descansa en una base sin fundamentos; es decir, aunque se da una explicitación evidente de la importancia de esta función para la institución museística, no se traduce necesariamente en un programa educativo bien estructurado y fundamentado en un proyecto educativo y, por tanto, se abandona al terreno de las intenciones y de los discursos. (Serrat, 2008, p. 168)

Conscientes de estos hechos, el AEAC y el GREID se proponen que el proyecto de investigación sea capaz de superar un planteamiento meramente procedimental de la educación artística, apostando claramente por la reflexión y la implicación del visitante:

... el camino desde el museo tradicional a un museo comprensivo es paralelo al recorrido realizado desde la enseñanza tradicional a la enseñanza renovada o comprensiva. De una insistencia en un carácter descriptivo de las disciplinas y de un afán taxonómico y enciclopedista se pasó a un énfasis en los procesos explicativos. De una actitud pasiva y receptiva no significativa, se ha pasado a un papel activo del observador o del aprendiz. De poner en marcha estrategias comunicativas basadas en el uso de conocimiento experto, se ha pasado a proponer una adecuación y una mediación entre el mensaje y los receptores generalmente novatos en dicho dominio específico. (Asensio 2002, citado por Serrat 2008, p.173)

No basta sólo con generar un taller o actividad educativa en el propio museo para todos los escolares. El reto vas más allá. Consiste en intentar que el alumnado y las escuelas dejen de ser meros visitantes, simples receptores de los programas y visitas que les ofrece el museo, para pasar a convertirse en auténticos protagonistas de la experiencia artística y cultural. Porque, como asegura Fontal $(2009,84)$, "hacer a los alumnos cómplices de una sensación, una idea o un sentimiento hacia un elemento artístico es la verdadera clave para que lo comprendan, lo compartan, lo sientan y lo disfruten". Por eso, no basta sólo con generar un taller o actividad educativa en el propio museo para todos los escolares.

2. En segundo lugar, la aplicación de las propuestas del Diseño Universal (DU) y la eliminación de las barreras, como mecanismos para aumentar la inclusividad en las ofertas que el Museo presenta a sus visitantes.

Los principios, las aplicaciones y la investigación sobre DU se han sido incorporando paulatinamente al diseño y preparación de entornos para la atención a las necesidades de las personas con discapacidad. Como establece la Convención Internacional de Naciones Unidas sobre los derechos de las personas con discapacidad, "por diseño universal se entenderá el diseño de productos, entornos, programas y servicios que puedan utilizar todas las personas, en la mayor medida posible, sin necesidad de adaptación ni diseño especializado. El diseño universal no excluirá las ayudas técnicas para grupos particulares de personas con discapacidad, cuando se necesiten." ${ }^{3}$ 
Se trata de abandonar un referente único (el alumno estándar, el visitante estándar, el ciudadano estándar), homogéneo y mayoritario, para pensar en las necesidades que puede experimentar cualquier persona. Los productos diseñados universalmente, o pensados teniendo en cuenta las necesidades de todos, facilitan el acceso con el máximo nivel de autonomía. Su objetivo es simplificar la vida de todos, haciendo que entornos, productos y comunicaciones sean más útiles para un mayor número de personas con un coste adicional bajo, o nulo.

3. En tercer lugar, las aportaciones Educación Inclusiva entendida como la educación que garantiza la presencia, la participación y el aprendizaje de todo el alumnado.

En opinión de Espinosa (2006) estos principios son la clave para la realización de un auténtico producto cultural accesible y el primer paso para eliminar todas las barreras para poder alcanzar la plena inclusión educativa y social: "Inclusión significa un cambio de mentalidad muy sencillo pero de enormes consecuencias: la marginación de los discapacitados no viene dada por sus respectivas discapacidades: la produce la sociedad cuando crea equipamientos no accesibles para ellos. Las barreras las creamos nosotros" (Espinosa, 2006, p.1)

Trabajar por y para la inclusión, aplicando los principios del DU, tanto en el ámbito escolar como en otros ámbitos y entornos sociales (como es el caso de los museos), supone la introducción de otro concepto básico: las barreras.

Páez y Delphine (2007, p.9), siguiendo el concepto acuñado por la OMS, definen las barreras como:

Todos aquellos factores ambientales que rodean una persona, que cuando están presentes o ausentes, limitan el funcionamiento y generan discapacidad. Entre ellos se incluyen aspectos tales como el ambiente físico inaccesible, la falta de tecnología asistencial adecuada, actitudes negativas de la población, así como servicios, sistemas y políticas que no existen o dificultan la participación de las personas con discapacidad en todas las áreas de la vida.

En definitiva, barreras son todos aquellos elementos presentes o ausentes en la sociedad, que imposibilitan o dificultan que cualquier persona pueda participar libre y plenamente, accediendo de manera independiente y personalizada a los espacios, la información y los contenidos.

Son muchas las clasificaciones de barreras que presentan los expertos en la materia. A modo de ejemplo Gómez del Águila (2012) clasifica las barreras en físicas, sensoriales, cognitivas y sociales. Oriol Ripoll $1^{4}$ presenta el concepto de barreras de la creatividad, dar por hecho las cosas, seguir estereotipos. Y, siguiendo a Páez y Delphine (2007) las barreras se clasifican en:

- Barreras arquitectónicas urbanísticas.

- Barreras arquitectónicas en los edificios

- Barreras en el transporte.

- Barreras en les comunicaciones sensoriales.

Tomando como referencia las premisas del DU, pretendemos que el proyecto de investigación que presentamos ayude a identificar las barreras existentes en el museo, de modo que sea posible garantizar el derecho de los alumnos con discapacidad, u 
otras condiciones de vulnerabilidad, a estar presentes, a aprender y a participar de la oferta cultural del museo, en condiciones de igualdad, con sus compañeros y compañeras sin estas circunstancias. Porque cada barrera que cae nos conduce hacia una sociedad más justa, más democrática e inclusiva.

\section{Hipótesis y objetivos de la investigación}

Partimos de la hipótesis de que si se analizan los espacios y las programaciones de las actividades del museo a partir de los principios de la escuela inclusiva y del DU, es posible optimizar las experiencias de aprendizaje que se producen en este espacio, aumentando las cotas de inclusión educativa y social.

Desde esta perspectiva, el museo no es un medio físico, sino un agente activo en el proceso de construcción de aprendizajes para todos y todas, independientemente de la edad, y de la capacidad cognitiva y física del visitante.

Para verificar dicha hipótesis, el estudio se articula en torno a dos objetivos básicos:

- Evaluar la accesibilidad del museo, centrándonos de manera especial en identificar las barreras de acceso al edificio, a la información y a los contenidos que ofrece el museo a cualquier visitante.

- Evaluar la accesibilidad de las visitas y talleres que el museo ofrece a las escuelas, identificando las barreras de acceso a la información, la participación y el aprendizaje que experimentan los grupos escolares.

\section{Metodología}

Para el logro de los objetivos planteados optamos por una metodología dirigida a la evaluación y análisis de la respuesta educativa (Stake, 2006), orientada hacia la toma de decisiones y la optimización de la práctica educativa.

Para la consecución del primer objetivo se utilizó la observación directa mediante el diseño y aplicación de un listado de control, estructurado en cuatro ámbitos de acuerdo con la clasificación de barreras que presentan Páez y Delphine (2007): el entorno físico del museo, la circulación interior, el acceso a la información y el acceso a los contenidos. Para cada uno de estos ámbitos se concretaron diferentes ítems de observación, tal y como queda recogido en el Anexo I.

Para alcanzar el segundo objetivo (evaluar la accesibilidad de las visitas y talleres destinados a las escuelas) se utilizaron las siguientes técnicas:

-

- Análisis de documentos referentes a las programaciones de las actividades del museo: memorias anteriores a la investigación, proyectos educativos del AEAC, dossier para los docentes previo a las visitas, etc.

- Grupo de discusión y seminario de formación con el fin de producir datos e ideas a través de la confrontación y el debate, así como para dar a conocer los resultados obtenidos.

- Registro en video de las visitas escolares para su posterior análisis. 
En total, se registraron en video 5 visitas de diferentes Centros de Educación Primaria e Infantil, un Instituto de ESO y un centro de Educación Especial de Mallorca.

\begin{tabular}{|c|c|c|}
\hline CENTRO & CURSO & VISITA-TALLER \\
\hline CP Camilo José Cela & Alumnado de P5 & Taller de Power Food \\
\hline CP Es Coll den Rabassa & Alumnado de P5 & Exposición de Kieffer \\
\hline CEE Mater Misericordiae & $16-21$ años & Taller Kieffer \\
\hline IES Damià Huguet & $14-16$ & Taller Power Food \\
\hline CEE Asnimo & Alumnado Adulto & Taller Power Food \\
\hline
\end{tabular}

Tabla 1: Relación de centros cuyas visitas al museo fueron grabadas en video para su posterior análisis

El procedimiento de análisis de las filmaciones en video fue diseñado para identificar las barreras que se surgían en el proceso de comunicación que se producía en las visitas y/o talleres. Se analizaron tanto las barreras de acceso a la información, las barreras a la participación en la actividad, así como también las barreras que dificultaban el aprendizaje de los participantes.

Se diseño una parrilla de recogida de información (Anexo II) y otra de análisis (Anexo III) que diferenciaba, siguiendo la estructura que presentaban las visitas-talleres, tres momentos básicos:

1. Acogida: Este momento recoge el acceso al museo, donde el monitor de la visita se presenta y saluda al grupo de alumnos.

2. Actividad: Es el momento que se desarrolla desde la presentación de las instrucciones y objetivos de la visita o taller, hasta la finalización de la misma.

3. Despedida: Momento de evaluación y cierre de la actividad.

\section{Fases de desarrollo de la investigación}

La primera fase fue la de constituir un equipo de trabajo formado por los investigadores del GREID y los miembros del AEAC del museo, con el objetivo de desarrollar un proceso de reflexión compartida sobre las acciones que estaba desarrollando el museo y la atención a las necesidades de sus visitantes.

En esta primera fase, se plantearon algunas de las preguntas siguientes, a partir de las cuales el proyecto de investigación iba adquiriendo forma: ¿Qué concepción tiene el AEAC sobre la función didáctica del museo y, especialmente, qué papel y objetivos atribuyen a las visitas-taller? ¿Cómo conciben las visitas-taller? ¿Qué actividades proponen a los alumnos? ¿Qué materiales ofrecen? ¿Cuál es la secuencia didáctica de la visita y a qué concepción educativa responde? ¿Cómo acogen al alumnado con necesidades educativas especiales (nee) en estas visitas?

Esta fase finalizó con la firma de un convenio entre las dos instituciones, estableciéndose el marco desde el cual proyectar y diseñar este primer estudio. 
En una segunda fase, el equipo realizó una búsqueda documental sobre didáctica y museos y sobre las experiencias museísticas existentes en nuestro país en relación con la accesibilidad de sus visitantes. Se encontraron experiencias muy interesantes en diversos museos nacionales e internacionales. ${ }^{5}$

También en esta segunda fase se concretaron los instrumentos que se iban a utilizar en el estudio para recoger y analizar la información que permitiera identificar barreras de acceso, de participación y de aprendizaje, tanto en el edificio como en las visitas-taller. Las decisiones que se acordaron fueron usar y/o crear los siguientes instrumentos:

a) El análisis de los documentos referentes a las programaciones de las actividades del museo: memorias anteriores a la investigación, proyectos educativos del AEAC, dossier para los docentes previo a las visitas, etc

b) La grabación en video de las visitas de los centros educativos al museo

c) El diseño de instrumentos de observación para la identificación de las barreras arquitectónicas del edificio (Anexo I)

d) La definición de parrillas para el análisis de los datos recogidos (Anexo II)

e) Concreción del grupo de discusión con los representantes del AEAC y el GREID para valorar los datos recogidos y llegar a propuestas de mejora.

Durante la tercera fase se llevó a cabo la recogida de datos a través de los instrumentos citados en la fase anterior.

La cuarta fase se dedicó al análisis de los datos recogidos con las parrillas diseñadas para tal efecto.

Y en la quinta fase se desarrollaron las acciones siguientes para valorar y difundir los resultados obtenidos:

a) Rediseño del protocolo de recogida de información de las características del grupo escolar previa a la visita al museo con el objetivo de ayudar en el Área de Educación a adaptar la visita a las necesidades que se requieren para de que todo el alumnado pueda aprender.

b) Presentación al personal del museo de los resultados del estudio de accesibilidad física y cognitiva obtenidos.

c) Jornada de Formación teórico práctica sobre competencias básicas y currículum escolar para los miembros del Grupo de investigación educativa en red (departamentos de educación de diferentes museos de España), para compartir y valorar el proceso llevado a cabo en Es Baluard.

\section{Resultados de la investigación:}

a) En relación al primer objetivo, identificación de las barreas de acceso que presenta el edificio, la información y los contenidos que ofrece el museo, vamos a prestar atención a las principales barreras detectadas ${ }^{6}$ :

b) En relación al entorno físico del museo, se detectan algunas rampas 
excesivamente largas y construidas con rejas metálicas que dificultan la circulación; la puerta de entrada es de cristal y no está señalizada a la altura adecuada.

c) En relación a la circulación interior, se constata que en las exposiciones algunas obras se colocan en el centro de la sala sin señalización en el suelo; otras, pueden resultar un obstáculo con el que chocar o caerse. Solo existe un ascensor con una numeración de las plantas que no coincide con la señalización que luego se da en cada una de ellas. La tienda presenta muchas barreras de acceso (mostrador excesivamente alto, pasillos estrechos, estanterías altas...). En el plan de evacuación no existe ningún responsable de las personas con discapacidad funcional. No hay ninguna señalización en braille.

d) En cuanto a la información, cabe destacar que no se ofrece en los diversos formatos accesibles (macro-caracteres, braille, información sonora...). La página web del museo tampoco es accesible por canales complementarios al canal visual. La aproximación física no está asegurada en todas las obras y muchos textos informativos se elaboran con letras adhesivas sobre cristales, lo que dificulta su lectura.

e) En cuanto al acceso a los contenidos, no hay ningún recurso para acceder de manera táctil a la información y no se cuenta con audio-descripciones, audioguías, textos de lectura fácil o cualquier otro tipo de apoyo.

En resumen, tal y como indican estos datos, no es suficiente con que el edificio respete los parámetros de accesibilidad en su edificación. Es necesario y urgente reclamar que tales parámetros se den también en el uso que se hace del edificio: el mobiliario de descanso, los carteles informativos, la página web, la distribución de las exposiciones, las vitrinas de la tienda...

La organización de las exposiciones temporales o de otros actos programados por el Museo, así como de su página web, son otros aspectos que también pueden rediseñarse aplicando los principios del DU.

En relación al segundo objetivo (evaluar la accesibilidad de las visitas y talleres dirigida a los escolares), se ponen de manifiesto que:

- En la fase previa a la visita, a partir del análisis documental de los documentos que el AEAC distribuye a las escuelas, se vislumbra que la propuesta didáctica que el museo ofrece a los escolares reproduce una secuencia didáctica centrada en la información que da el monitor y con un papel pasivo por parte del alumnado y del profesorado; que los docentes utilizan el museo de forma muy restringida y en la visita asumen un papel pasivo, y que en las visitas/ talleres no se contemplan adaptaciones o apoyos para el alumnado con algún tipo de necesidad educativa especial.

- En la hoja de instrucciones que se da a las escuelas recordando las normas de funcionamiento de la visita, se detecta la ausencia de información sobre los objetivos que se van a trabajar en cada una de las etapas de la sesión y ante cada obra.

- Cabe destacar que no se mantiene una reunión previa a la visita con el 
profesorado donde poder recoger aquellas informaciones útiles y necesarias para mejorar y adaptar la visita o el taller. Nos referimos a la descripción del grupo de alumnos que participará en la actividad, para conocer qué tipo de ayudas necesitan para dibujar, explicar, andar, hablar, participar...Se detecta también la ausencia de este intercambio de opiniones con el profesorado tan necesario para poder saber cuáles son las dinámicas que se siguen en clase, de qué modo se ha preparado la visita, que sentido se le ha dado... incrementando así las posibilidades de adaptar la información que se transmite desde el museo a las experiencias y conocimientos previos de los escolares.

Por otra parte, del análisis de la información sobre las barreras detectadas en los diferentes momentos de las actividades, destacan los siguientes resultados:

a) Durante la Acogida:

- La recepción y bienvenida al museo corre a cargo del monitor en la mayoría de las visitas registradas; en ningún caso la entrada al edificio se hace por parejas o toma protagonismo el alumnado. Por eso, el alumnado que utiliza silla de ruedas entra sólo (con un adulto de apoyo) y lo hace siempre en último lugar.

- Más allá del primer contacto inicial, el monitor no otorga un significado didáctico a este momento: no se explica la estructura que tendrá la visita, ni sus partes, ni los objetivos que se trabajarán en cada una de ellas.

- Curiosamente, sólo se comunican y esclarecen cuáles son las normas de comportamiento en el interior del museo al alumnado procedente de los institutos de secundaria.

b) Durante el Desarrollo de la Actividad:

- En las visitas a las exposiciones grabadas en video se constata el hecho de que el alumnado pasa la mayor parte del tiempo sentado, escuchando o en silencio. Este es, por otra parte, el papel demandado por el monitor, quien asume todo el protagonismo al dedicarse casi de manera exclusiva a la explicación de las diferentes obras.

- No se da la opción a que sea el alumnado quien decida ante qué cuadro, fotografía o escultura va a detenerse el grupo. Esta decisión la toma también el monitor. Por otra parte, cuando el monitor detiene al grupo para que observe con detalle un elemento, no da ninguna explicación sobre el objetivo que se persigue con la observación.

- No se trabajan las hipótesis de interpretación del cuadro (¿qué nos quiere decir el autor?, ¿qué título podrías al cuadro?) a partir de preguntas abiertas para dar cabida a diversas opciones de respuesta.

- Otras barreras detectadas guardan relación con el hecho de que no se llevan a cabo actividades ante la obra observada, ni se deje un período de tiempo suficiente para que el alumnado observe en silencio, o proceda a contestar 
o formular preguntas. En ninguna de las sesiones se invita al alumnado a intercambiar sus opiniones, sensaciones, reflexiones con los compañeros del grupo. No se da tiempo para la exploración libre de la sala (a pesar de que el tamaño del grupo es reducido), para que el alumnado descubra su espacio y contenido.

- Las preguntas, formuladas mayoritariamente por el monitor, son de carácter cerrado y sirven sobre todo para comprobar que el alumnado sigue más o menos la explicación. En ninguna sesión, se detectan preguntas relacionadas con la dimensión emocional del alumnado: ¿qué sentís ante este cuadro?, ¿qué emociones os provoca?, ¿qué impacto?, ¿qué recuerdos?, ¿qué sensaciones?, ¿os recuerda alguna película, canción, experiencia...?

- En las visitas no se trabaja activando todos los sentidos (aprendizaje multisensorial) ni se crea la necesidad de formularse preguntas sobre lo que se percibe. Muchas de las actividades propuestas reproducen materiales y recursos que también se usan en las escuelas. De hecho, no se aprovecha el espacio y los recursos del museo para simular, por ejemplo, un contexto diferente y hacer actividades que por falta de espacio no pueden desarrollarse en los centros escolares.

- Por otra parte, los videos que acompañan las visitas/talleres no están adaptados para el alumnado con déficit visual y auditivo, lo cual supone una importante barrera para su aprendizaje y participación.

- Finalmente, se detecta que el protagonismo de la actividad recae en el monitor, quien establece una relación unidireccional con el grupo clase. En los momentos de debate, no se utilizan las posibilidades del espacio y los contenidos que nos brinda el museo.

c) Despedida:

- En el momento de finalizar la actividad, no se destina un espacio de tiempo para que el alumnado reflexione y explique qué ha aprendido, qué le ha gustado más... Tampoco se da ningún tipo de elemento que sirva de recuerdo o propuesta para seguir investigando y trabajando con posterioridad la temática en la escuela.

Cabe mencionar que la presentación y discusión de estos resultados en la quita fase del estudio, permitió llegar a los siguientes acuerdos y propuestas de intervención:

- Respecto al documento creado para la recogida inicial de información de los grupos escolares, es necesario saber los conocimientos previos con los que llega cada grupo (lo que han trabajado en la escuela / aula)

- Se redactarán las normas de comportamiento a seguir en el museo para que sean conocidas previamente a la visita en los centros escolares, siempre en positivo, como un listado de valores.

- Se llevará a cabo una revisión, reducción y estructuración de los objetivos y 
los contenidos de las programaciones presentadas por el AEAC, teniendo en cuenta que sean evaluables y tengan en cuenta criterios inclusivos.

A su vez, la presentación de los resultados a todo el personal del museo, acompañada de alternativas posibles, promovió la eliminación de algunas de las barreras identificadas, empezando por las que suponían un coste cero, gracias a la colaboración de entidades y asociaciones de personas con diversidad funcional.

A modo de ejemplo, se anotó la posibilidad de subtitular los audiovisuales, las conferencias, formar a algún trabajador en la traducción bimodal, ofrecer la posibilidad de acceder a la información en braille, en macro-caracteres, disponer de mapas de la distribución del museo y las obras que se pueden visitar, permitir el acceso táctil de diferentes obras, etc. Una vez más se evidenció la diferencia entre garantizar la accesibilidad y fomentar la inclusión

\section{Conclusiones de la investigación}

El estudio permite comprobar la hipótesis inicial, afirmando que los principios de la escuela inclusiva y del DU son un buen instrumento para analizar y optimizar los espacios, las actividades y las experiencias de aprendizaje que se producen en el Museo.

Recogiendo los dos objetivos básicos de la investigación, los resultados apuntan la existencia de una gran diversidad de barreras, tanto en el acceso al edificio, a la información, a la posibilidad de participar y sobretodo de aprender que ofrece el museo a cualquier visitante.

Con el trabajo compartido entre los profesionales del AEAC, llegamos a la conclusión final de que el museo, con sus espacios, sus dimensiones y su patrimonio, puede facilitar de manera única proyectos interdisciplinares y experiencias integrales (cognitivas, afectivas y relacionales) ayudando a la escuela a ampliar y trascender sus propias fronteras. Es decir, el Museo ofrece la posibilidad de enseñar y aprender de manera diferente, con todos los sentidos y convirtiendo el espacio en un agente más del proceso educativo ${ }^{7}$. Por ello, es necesario establecer puentes entre ambas instituciones (escuela y museo; museo y escuela) con el objetivo de crear experiencias que faciliten el acceso, la participación y el aprendizaje de todos y todas, sin exclusiones, y superando cualquier acto que ponga barreras a la creatividad.

\section{Discusión y planteamientos para futuras investigaciones}

Uno de los aspectos más relevantes del estudio es el análisis de la función de los profesionales del área de educación del museo y de la escuela, con el objetivo de intentar dar respuesta a la demanda de mejorar la relación entre ambas instituciones, para optimizar las experiencias formativas que se comparten.

Recogiendo las palabras de Huerta (2010) "son muchos los estudios y los autores y autoras que en los últimos años se han preocupado por la educación en museos (...), pero a pesar del creciente interés por investigar estas cuestiones, la formación de los educadores en materia de educación artística sigue resultando insuficiente". 
Consideramos que debe de puntualizarse que esta falta de formación se da en ambas instituciones, ya que en la escuela hemos podido evidenciar la falta de conocimiento sobre el potencial de aprendizaje que presentan los espacios, sobre todo si se comparte el trabajo a desarrollar en ellos, sea el museo o el aula.

A modo de ejemplo, observamos que durante la visita o taller, el profesorado asume un papel pasivo, de simple espectador o acompañante. Sólo participa seleccionando la actividad de entre el listado que le ofrece el museo.

Compartimos así las aportaciones del grupo de investigación Arte y Educación de la Universidad de Valencia, al anotar que:

- El lenguaje de los docentes está ambientado en las prácticas pedagógicas curriculares.

- Que dichos colectivos necesitarían de un registro común que permitiese un acercamiento entre ambos.

- Que las TIC deberían utilizarse para reforzar dicho registro común.

- Que todo resultaría mucho más eficiente en las visitas a museos si se tuviesen en cuenta los puntos de encuentro de ambos colectivos de trabajadores culturales.

- Que para el alumnado a quien va dirigida la visita al museo resultaría mucho más productiva y emotiva la visita si la actividad participase de sus vivencias. (Huerta, 2010, p.71).

Son muchas las preguntas que nos formula la realidad estudiada, de entre las que destacamos: ¿por qué compartiendo los mismos objetivos, museo y escuela, no aprovechamos ambos espacios para crear experiencias que realmente favorezcan el aprendizaje de todos los visitantes?

Este es el reto que nos planteamos y que con los seminarios de formación intentamos trabajar. Asumimos la limitación de que estas acciones formativas, una vez más, las hemos empezado a desarrollar de manera separada, es decir, seminarios con educadores en el museo y seminarios con docentes en las escuelas, en vez de crear grupos mixtos donde intentar romper con estas diferencias de registro.

Aún así, apostamos por la creación de espacios compartidos de formación, abiertos, interinstitucionales e inclusivos, con el objetivo de trabajar para el desarrollo de ciudadanos y ciudadanas con más competencia artística y cultural.

\section{Referencias}

AA.VV. (2010). Arte Contemporáneo y Educación Especial. Huesca: Ajuntament de Lleida, Centre d'Art La Panera, CDAN. Centro de Arte y Naturaleza. Fundación Beulas y Fundació Es Baluard Museu d'Art Modern i Contemporani de Palma. Asensio, M. y Pol, E. (2002) Nuevos escenarios en educación. Aprendizaje informal sobre el patrimonio, los museos y la ciudad. Buenos Aires, Aique Grupo Editor. Cifre, E. (2011). Es Baluard, la educación y su relación con el ámbito hospitalario. Primeras experiencias. Arte, Individuo y Sociedad. Vol. 23 Núm. Especial, 229244. 
Espinosa Ruiz, A. (2006). El concepto de inclusión en programas intrerpretativos en museos. Trabajo presentado en la V Jornada AIP, Navarra.

Fontal Morillas, Olaia (2009). Los museos de arte: un campo emergente de investigación e innovación para la enseñanza del arte. REIFOP, 12 (4), 75-88. Recuperado de http://www.aufop.com/

García del Dujoy, Á. y Muñoz Rodríguez, J.M. (2007). Pedagogíade los espacios análisis terminológico y construcción conceptual. Ethos Educativo.39.p.7.

Gómez del Águila (2012). Accesibilidad e Inclusión en espacios de Arte: ¿cómo materializar la utopía? Arte, Individuo y Sociedad. Vol. 24 (1), 77-90.

Hernández, (2006). Planteamientos teóricos de la museología. Gijón: Ediciones Trea

Huerta, R. (2011). Maestros, museos y artes visuales. Construyendo un imaginario educativo. Arte, Individuo y Sociedad, 23 (1), 55-73.

Jové Moncús, G. y Betrián Villas, E. 2012: "Entretejiendo encajes" entre la universidad, los centros de arte y las escuelas. Arte, Individuo y Sociedad, 24 (2), 301-314

Martínez (2008). Sobre la participación de los públicos en la configuración de actividades del Museo Nacional Centro de Arte Reina Sofía. (En construcción). Recuperado de http://edumuseos.blogspot.com.

Muntaner, Forteza, Rosselló, Verger y De la Iglesia, (2010). Estándares e indicadores pera analizar la calidad de vida del alumnado con discapacidad durante su proceso educativo. Palma de Mallorca: Edicions UIB

Padró, C. (2003). La museología como forma de reflexionar sobre los museos como zonas de conflicto e intercambio. En J.P. Lorente, Museología critica y arte contemporáneo. Zaragoza: Prensa Universitaria de Zaragoza.

Páez, G. i Delphine, P. (2007) La Accesibilidad de los Teatros: El Caso del Teatro Valle-Inclán de Madrid. Tesina inédita. Máster en Gestión Cultural, Universidad Complutense de Madrid.

Santacana Mestre, J. y Hernández Cardona, F.X. (2006). Museología crítica. Gijón: Ediciones Trea

Serrat Antolí, N. (2008). Accions didàctiques en el marc dels museus. Una investigación entre el museu i l'escola. Temps d'Educació, 34, 167-182.

Stake, R.E.(2006). Multiple Case Study Análisis. New Cork: Guilford Press. 


\section{Anexo I.}

Instrumento de observación de las barreras arquitectónicas del edificio.

\begin{tabular}{|l|l|l|l|}
\hline EVALUACION DE LA ACCESIBILIDAD & \multicolumn{1}{|l|}{} \\
\hline 1. ENTORNO FÍSICO DEL MUSEO & SI & NO & OBSERVACIONES \\
\hline 1.1. Transporte público accesible & & & \\
\hline 1.2. Plazas de aparcamiento protegido & & & \\
\hline 1.3. Señalización de la llegada al centro & & & \\
\hline $\begin{array}{l}\text { 1.4. Rampas, pavimentos e itinerarios } \\
\text { exteriores }\end{array}$ & & & \\
\hline 1.5. Circulación en las entradas y salidas & & & \\
\hline 2. CIRCULACION INTERIOR & & & \\
\hline 2.1. Itinerarios y puertas & & & \\
\hline 2.2. Ascensores, escaleras y rampas interiores & & & \\
\hline 2.3. Paredes y tablones & & & \\
\hline 2.4. Señalización e iluminación & & & \\
\hline 2.5. Lavabos & & & \\
\hline 2.6. Tienda, oficinas, restaurante y auditorio & & & \\
\hline 2.7. Terrazas & & & \\
\hline 2.8. Personal del museo & & & \\
\hline 2.9. Mobiliario de descanso & & & \\
\hline 2.10. Plan de evacuación & & & \\
\hline 3. ACCESO A LA INFORMACIÓN & & & \\
\hline 3.1. En la página web & & & \\
\hline 3.2. En las visitas guiadas & & & \\
\hline 3.3. En los carteles y paneles informativos & & & \\
\hline 3.4. En las guías, los dípticos y los catálogos & & & \\
\hline 3.5. En la biblioteca & & & \\
\hline 4. ACCESO A LOS CONTENIDOS & & & \\
\hline 4.1. En las obras y las reproducciones & & & \\
\hline 4.2. En los audiovisuales, charlas... & & & \\
\hline 4.3. En las audio-descripciones & & & \\
\hline 4.4. En las visitas se cuenta con signo-guía & & & \\
\hline
\end{tabular}




\section{Anexo II.}

Parrilla para la identificación de las barreras que se dan durante las actividades.

\begin{tabular}{|l|l|}
\hline $\begin{array}{l}\text { BIENVENIDA. (Este momento recoge el acceso al museo, donde el monitor de la } \\
\text { visita se presenta y saluda al grupo de alumnos). }\end{array}$ \\
\hline ¿Cómo se da la bienvenida? \\
\hline $\begin{array}{l}\text { ¿Cómo es el ritual o saludo? ¿Se personaliza de algún } \\
\text { modo? }\end{array}$ & \\
\hline $\begin{array}{l}\text { ¿Se utiliza algún sistema de apoyo o de carácter } \\
\text { organizativo para facilitar una entrada pausada y } \\
\text { tranquila? }\end{array}$ & \\
\hline ¿Se explican los objetivos y la estructura de la visita? & \\
\hline $\begin{array}{l}\text { ¿Se recuerdan/comprueban las normas de } \\
\text { comportamiento? }\end{array}$ & \\
\hline
\end{tabular}

\section{Despedida}

Se da la oportunidad al alumnado para que reflexione sobre los aprendizajes realizados.

Se recogen evidencias del aprendizaje realizado por el alumnado y las valoraciones del profesorado.

Se establece algún compromiso de seguimiento del trabajo realizado en el museo.

\section{VISITA EXTERIOR: LAS MURALLAS/VISITA INTERIOR: LA EXPOSICIÓN}

\begin{tabular}{|l|l|}
\hline ¿Cómo es la secuencia general? & \\
\hline ¿Cuánto dura la explicación del monitor? & \\
\hline $\begin{array}{l}\text { ¿Qué información da? ¿Qué estrategias comunicativas } \\
\text { utiliza? }\end{array}$ & \\
\hline $\begin{array}{l}\text { ¿Qué preguntas realiza? ¿parte de los conocimientos } \\
\text { previos de los alumnos? }\end{array}$ & \\
\hline ¿Cómo gestiona la participación de los alumnos? & \\
\hline $\begin{array}{l}\text { ¿Utiliza algún recurso o apoyo para la personalización? } \\
\text { ¿Se utilizan diferentes materiales? }\end{array}$ & \\
\hline
\end{tabular}




\begin{tabular}{|l|l|}
\hline TALLER & \\
\hline $\begin{array}{l}\text { ¿Qué instrucciones se da para iniciar el } \\
\text { trabajo? }\end{array}$ & \\
\hline ¿Cuál es la propuesta de actividades? & \\
\hline ¿Cuál es la propuesta de materiales? & \\
\hline $\begin{array}{l}\text { ¿Se utiliza alguna estrategia de } \\
\text { personalización? }\end{array}$ & \\
\hline ¿Se utiliza algún apoyo o recurso especial? & \\
\hline
\end{tabular}

\section{Anexo III.}

Parrilla de análisis de las barreras de información, participación y aprendizaje que se dan en las visitas y/o talleres del museo.

\begin{tabular}{|l|l|l|l|}
\hline & ACOGIDA & ACTIVIDAD & DESPEDIDA \\
\hline BARRERAS INFORMACIÓN & & & \\
\hline BARRERAS PARTICIPACIÓN & & & \\
\hline BARRERAS APRENDIZAJE & & & \\
\hline
\end{tabular}

\section{Notas}

- Este trabajo se desarrolla en el marco del proyecto de investigación SEJ-200506467/EDUC, titulado: "Estándares e indicadores para analizar la calidad de vida del alumnado con nee asociadas a discapacidad en su proceso educativo", financiado por el Ministerio de Educación y Ciencia, y llevado a cabo por el GREID (Grupo de Investigación en Escuela Inclusiva y Diversidad) de la Universitat de les Illes Balears.

- Experiencia similar de colaboración la encontramos en Jové Moncús, G. y Betrián Villas, E. 2012: "Entretejiendo encajes" entre la universidad, los centros de arte y las escuelas. Arte, Individuo y Sociedad, 24 (2), 301-314

- Artículo 2 de la Convencion Internacional de Naciones Unidas sobre los derechos de las personas con discapacidad. Recuperado de http://www.feaps.org/ archivo/centro-documental/cat_view/86-derechos.html

- Conferència inaugural de les XV Jornades de Museus i Educació del Museu Marítim de Barcelona, celebrades els dies 23 i 24 de febrer de 2012

- Véase los resultados del proyecto en "Intercambio entre programas de educación especial de centros de arte contemporáneo de la Eurorregión PirineosMediterráneo”. En AAVV. Arte Contemporáneo y Educación Especial. 
- Huesca: Ajuntament de Lleida, Centre d'Art La Panera, CDAN. Centro de Arte y Naturaleza. Fundación Beulas y Fundació Es Baluard Museu d'Art Modern i Contemporani de Palma, 2010.

- Resultados obtenidos a partir del Trabajo Final de Máster inédito llevado a cabo por la participación de Esperanza Marimón Mut del, bajo el título L'accessibilitat al Museu d'Art Modern i Contemporani de Palma, Es Baluard.

- La pedagogía de los espacios es una línea de investigación que propone incorporar y considerar los espacios como agentes primordiales en el proceso educativo". En García del Dujoy, Á. y Muñoz Rodríguez, JM. (2007). Pedagogía de los espacios análisis terminológico y construcción conceptual. Ethos Educativo.39.p.7. 